\title{
Inhibitory effects of orally administrated liposomal bovine lactoferrin on the LPS-induced osteoclastogenesis
}

\author{
Eizo Yamano ${ }^{1,2}$, Mutsumi Miyauchi ${ }^{1}$, Hisako Furusyo ${ }^{1}$, Aki Kawazoe ${ }^{2}$, Atsushi Ishikado ${ }^{3}$, Taketoshi Makino ${ }^{3}$, \\ Kazuo Tanne ${ }^{2}$, Eiji Tanaka ${ }^{4}$ and Takashi Takata ${ }^{1}$
}

Bovine lactoferrin (bLF) modulates the production of proinflammatory cytokines including tumor necrosis factor (TNF)- $\alpha$, and may thus control alveolar bone destruction associated with periodontitis. In this study, the effects of bLF on mRNA expression in lipopolysaccharide (LPS)-stimulated osteoblasts (OBs) and on LPS-induced osteoclastogenesis were examined. The inhibitory effects of oral administration of liposomal-bLF (L-bLF), which improved the robustness of bLF to digestive enzymes, on alveolar bone resorption using LPS-induced periodontitis rat model are also reported. Three groups of 7-week-old male Wistar rats were treated with L-bLF (L-bLF group), bLF (bLF group), or the vehicle (control group) in drinking water ( $n=6$ in each group). On day 7, LPS was topically applied into the gingival sulcus. Number of osteoclasts and immunoexpression of TNF- $\alpha$ were analyzed. The bLF inhibited the upregulation of TNF- $\alpha$-mRNA- and upregulation of receptor activator of $\mathrm{NF} \kappa \mathrm{B}$ (RANKL)-mRNA expression and eliminated downregulation of osteoprotegerin (OPG)-mRNA expression in LPS-stimulated OBs and reduced LPS-induced osteoclastogenesis in co-culture with primary OBs and bone marrow cells. In the control group, the number of osteoclasts increased after LPS treatment. The number of osteoclasts that appeared along the alveolar bone margin was significantly reduced $(P<0.01)$ in the L-bLF but not in the bLF group. Furthermore, L-bLF suppressed upregulation of TNF- $\alpha$ immunoexpression in periodontal tissue and TNF- $\alpha$ and interleukin (IL)-1 $\beta$-mRNA level in gingival tissue. The results of this study indicate that oral administration of L-bLF significantly reduces alveolar bone resorption induced by LPS stimulation through inhibition of TNF- $\alpha$ production and modulation of RANKL/OPG balance in OBs. It is suggested that L-bLF could be a potent therapeutic and preventive agent for attenuating alveolar bone destruction in periodontitis patients.

Laboratory Investigation (2010) 90, 1236-1246; doi:10.1038/labinvest.2010.80; published online 26 April 2010

KEYWORDS: bovine lactoferrin; liposomalization; LPS; osteoclast; proinflammatory cytokines; TNF- $\alpha$

Superficial periodontal tissues, an initial barrier to bacterial challenge, are constantly exposed to plaque-associated bacteria and bacterial lipopolysaccharide (LPS), which can induce an inflammatory reaction. ${ }^{1,2}$ Although inflammation is an essential component of the host defense response to bacterial challenge, excessive cytokine production from host cells resulted in consequent periodontal tissue destruction. Cytokines are rapidly synthesized and increased levels of proinflammatory cytokines, such as tumor necrosis factor
(TNF)- $\alpha$, interleukin (IL)-1 $\beta$, and IL-6, are detected in the inflamed gingival tissue and gingival crevicular fluid at inflamed site. ${ }^{3-5}$ It is well demonstrated that these proinflammatory cytokines having bone resorbing activity, act osteoblasts (OBs) and stimulate osteoclastogenesis through upregulation of receptor activator of $\mathrm{NF} \kappa \mathrm{B}(\mathrm{RANKL})$ and downregulation of osteoprotegerin (OPG) in OBs. ${ }^{6}$

Earlier, we have reported that initial periodontal tissue damage was provoked by topical application of Escherichia

\footnotetext{
${ }^{1}$ Department of Oral and Maxillofacial Pathobiology, Hiroshima University Graduate School of Biomedical Sciences, Minami-ku, Hiroshima, Japan; ${ }^{2}$ Departments of Orthodontics and Craniofacial Developmental Biology, Hiroshima University Graduate School of Biomedical Sciences, Minami-ku, Hiroshima, Japan; ${ }^{3}$ Health Care R\&D Division, Sunstar, Osaka, Japan and ${ }^{4}$ Department of Orthodontics and Dentofacial Orthopedics, University of Tokushima Graduate School of Oral Sciences, Minami-ku, Hiroshima, Japan

Correspondence: Dr T Takata, DDS, PhD, Department of Oral and Maxillofacial Pathobiology, Hiroshima University Graduate School of Biomedical Sciences, 1-2-3 Kasumi, Minami-ku, Hiroshima 734-8553, Japan.

E-mail: ttakata@hiroshima-u.ac.jp

Received 11 September 2009; revised 9 February 2010; accepted 24 February 2010
} 
coli LPS into the rat gingival sulcus; infiltration of numerous polymorphonuclear leucocytes in the junctional epithelium $(\mathrm{JE}),^{7}$ transient accumulation of exudative macrophages, ${ }^{8}$ vascular dilatation and inflammatory edema in the sub$\mathrm{JE}$, enhancement of proliferative activity of JE cells, ${ }^{9}$ and stimulation of osteoclastic bone resorption. ${ }^{7}$ Furthermore, using this animal model, we have shown upregulation of immunoexpression of TNF- $\alpha$, IL- $1 \beta,{ }^{10}$ and chemokines ${ }^{11}$ in periodontal tissue and suggested that transiently produced cytokines from the host cells may be involved in the initiation of inflammation and subsequent periodontal tissue destruction. Therefore, we consider that appropriate regulation of cytokine production by natural immunomodulator might be useful in reducing periodontal tissue destruction such as alveolar bone resorption.

Bovine lactoferrin (bLF), an iron-binding glycoprotein of the transferrin family with a wide spectrum of biological activities, can modulate the inflammatory response by binding potentially toxic-free iron. ${ }^{12}$ bLF may also affect immunological functions by modulating the production of cytokines TNF- $\alpha$, IL- $1 \beta$, and IL-6, by regulating natural killer cell activity, inhibiting antibody synthesis, and maturation of T cells. ${ }^{13-15}$ Although, the mechanism of bLF modulation of cytokine production is not well understood, this could be important locally at the site of inflammation, such as in the rheumatoid joint or in the periodontal tissue of the periodontitis. ${ }^{16-18}$ In fact, bLF has been shown to bind 'free' iron in synovial fluid and to reduce inflammation when injected intraarticularly in mice with collagen-induced arthritis. ${ }^{17}$ In addition, bLF is of food origin and has high safety. Therefore, we hypothesized that oral administration of bLF may be preventive or be a useful therapeutic treatment for periodontitis. However, bLF is digested by the gastric juice and thus only $60 \%$ of the intact bLF enters the small intestine during oral administration. ${ }^{19}$ Thus, specific enteric coating or specific delivery systems for bLF are needed for its effective oral administration. Liposomes prepared from naturally occurring biodegradable and nontoxic lipids have been proposed as efficient carriers for local delivery of therapeutic agents. Liposomalization of bLF has been shown to enhance the anti-inflammatory effects of bLF. ${ }^{20,21}$ Therefore, we stabilized bLF by liposomalization ${ }^{22}$ to enhance its absorbability from the intestinal lumen and transport into the blood circulation.

In this study, using various in vitro assays, we have shown that bLF inhibited LPS-induced osteoclastogenesis through reduction of TNF- $\alpha$ and RANKL expression and elimination of OPG suppression in osteoblastic cells with LPS stimulation. Moreover, in vivo study showed that orally administrated liposomal-bLF (L-bLF) significantly reduced alveolar bone resorption through the suppression of TNF- $\alpha$ locally produced by host cells with LPS stimulation. These results indicate the possibility of the preventive and therapeutic usage of bLF for LPS-induced periodontitis.

\section{MATERIALS AND METHODS Reagents}

The bLF was purchased from Morinaga Milk Industry (Tokyo, Japan) and L-bLF was prepared as described earlier. ${ }^{22}$ LPS from Aggregatibactor Actinomycetemcomitance (ATCC29522 strain) (A.a.-LPS) was kindly provided by Professor Tatsuji Nishihara of the Kyusyu Dental College.

\section{Cell Line and Cell Culture}

Primary OBs, obtained from mouse calvariae, ST2 (a bonemarrow-derived osteogenic cell line) cells and MC3T3-E1 cells were maintained in a minimum essential medium alpha medium ( $\alpha$-MEM) (Invitrogen, NY, USA) with $10 \mathrm{mM}$ HEPES (pH 7.2), 10\% FBS (Invitrogen) and $100 \mathrm{U} / \mathrm{ml}$ Penicillin-Streptomycin (Invitrogen) at $37^{\circ} \mathrm{C}$ in a humidified atmosphere of $5 \% \mathrm{CO}_{2}$.

\section{Gene Expression Experiments}

Expression of $L F$ receptors in osteoblastic cells

$\mathrm{OB}, \mathrm{ST} 2$, and MC3T3-E1 cells were plated in $60 \mathrm{~mm}$ culture dishes $\left(1 \times 10^{6}\right.$ cells/dish $)$ and cultured in $\alpha$-MEM containing $10 \%$ FBS and harvested after 2 days.

Effects of bLF on gene expression of osteoclastogenesis-related cytokines in ST2 cells with LPS stimulation

ST2 cells were seeded in $60-\mathrm{mm}$ culture dishes $\left(1 \times 10^{6}\right.$ cells/ dish) and cultured in $\alpha$-MEM containing 10\% FBS for 2 days. After incubation in fresh medium for 1 day, the cells were incubated with A.a.-LPS $(100 \mathrm{ng} / \mathrm{ml})$ with or without 2-hpretreatment with bLF $(10 \mu \mathrm{g} / \mathrm{ml})$. The cultured cells were harvested after $0,2,4,6,12$, and $24 \mathrm{~h}$ after LPS stimulation.

\section{$R N A$ extraction and RT-PCR analyses}

Total RNA was extracted from the harvested cells and gingival tissue using TRIzol reagent (Invitrogen). A measure of $1 \mu \mathrm{g}$ of total RNA was used for cDNAs synthesis using Rever Tra Dash (TOYOBO, Osaka, Japan) kit. Aliquots of total cDNA were amplified using KOD-Plus-DNA Polymerase (TOYOBO) and specific primer pairs (see Supplementary Table 1). Amplification was performed using a MyCycler thermal cycler (BIO-RAD, Tokyo, Japan). PCR products were electrophoresed on $1.5 \%$ agarose gels at $100 \mathrm{mV}$ and visualized by ethidium bromide.

Quantitative real-time RT-PCR was performed in the Light Cycler System (light cycler quick system 350S, Roche Diagnostics GmbH, Mannheim, Germany) using LightCyclerFastStart DNA Master SYBR Green I (Roche Diagnostics $\mathrm{GmbH}$ ) and specific primers for TNF- $\alpha$ and IL-1 $\beta$ (see Supplementary Table 1). Reaction product was quantified with GAPDH as the reference gene.

\section{Endocytosis of bLF by OBs}

ST2 cells were seeded in well-chamber slides and grown to subconfluence and bLF $(10 \mu \mathrm{g} / \mathrm{ml})$ was added and at $0,2,4$, $8,24 \mathrm{~h}$ after bLF addition, cells were fixed with $2 \%$ buffered 
formalin. Intracellular localization of bLF was determined by immunohistochemistry using the high polymer (HISTOFINE simple stain, NICHIREI, Tokyo, Japan) method. After incubation in $0.3 \%$ hydrogen peroxide in methanol for $60 \mathrm{~min}$ at room temperature to quench endogenous peroxidase activity, the culture cells were treated with protein block (DAKO, Carpinteria, CA, USA) for $30 \mathrm{~min}$ at room temperature. They were incubated with 1:100 dilution of monoclonal anti-bLF antibody (Hycult Biotechnology B.V., Netherlands) at $4{ }^{\circ} \mathrm{C}$ overnight. After twice in phosphatebuffered saline (PBS) for 5 min each, sections were incubated with HISTOFINE simple stain MAX-PO for $30 \mathrm{~min}$. The color was developed with $0.025 \% 3,3^{\prime}$-diaminobenzidine tetrahydrocloride in TRIS-HCC buffer plus hydrogen peroxide (DAKO) and counterstained with hematoxylin, dehydrated, cleared, and mounted.

\section{Cell Preparation and Osteoclast Formation Assay}

Primary OBs were obtained from calvariae of newborn ddY mice by conventional methods using collagenase. Bone marrow cells (BMCs) were collected from the femora and tibiae of 6- to 9-week-old male mice. Primary OBs $\left(1 \times 10^{4}\right.$ cells) and BMCs $\left(2 \times 10^{5}\right.$ cells $)$ were co-cultured for 5 days in $\alpha$-MEM containing $10 \%$ FBS in 96 -well tissue culture plates and incubated with A.a.-LPS $(1 \mu \mathrm{g} / \mathrm{ml})$ for the final 3 days. Some co-cultures were pretreated with or without bLF ( 1 or $10 \mu \mathrm{g} / \mathrm{ml})$. Co-cultures were then fixed and stained for tartrate-resistant acid phosphatase (TRAP; a marker enzyme of osteoclasts). All TRAP-positive cells with more than three nuclei or with less than two nuclei appeared in each well were counted under a microscope separately as osteoclast and preosteoclasts, respectively.

\section{Experimental Animals}

The experimental protocol described below was approved by the animal care committee of the Hiroshima University. A total of 126, 7-week-old (about 215g), male Wistar rats were kept at constant ambient temperature $\left(22^{\circ} \mathrm{C}\right)$ and fed a solid diet ad libitum. Rats were randomly divided into three groups $(n=42 /$ group $)$ and treated with either $10.0 \mathrm{~g} / \mathrm{l}$ L-bLF including $2.125 \mathrm{~g} / \mathrm{l} \mathrm{bLF}$ and $7.08 \mathrm{~g} / \mathrm{l}$ maltitol as a stabilizer (L-bLF group), $2.125 \mathrm{~g} / \mathrm{l}$ non-L-bLF and $7.08 \mathrm{~g} / \mathrm{l}$ maltitol (bLF group) or $7.08 \mathrm{~g} / \mathrm{l}$ maltitol (control group) in drinking water, for 7 days before LPS application throughout the experiments. Body weights and water consumption were recorded.

\section{Application of LPS}

Rats were treated with LPS on day 7 of bLF treatment. Under intraperitoneal anesthesia with $10 \%$ pentobarbital sodium (Somnopentyl:0.6 ml/kg: Schering-Plough Animal Health, USA), rats were fixed on his back on an experimental stand. A cotton roll (2 $\mathrm{mm}$ diameter and $1 \mathrm{~cm}$ in length) saturated with $5 \mathrm{mg} / \mathrm{ml}$ E. coli LPS (Sigma Chemical, St Louis, MO, USA) in sterile physiological saline (Otsuka Med., Tokyo, Japan) was placed parallel to the marginal periodontal tissues in the right and left molar regions for $1 \mathrm{~h}$. The cotton roll was changed every $20 \mathrm{~min}$. Six rats from each group were killed at 0,1 , or $3 \mathrm{~h}$, or $1,2,3$, or 7 days after the LPS treatment by an overdose of ethyl ether.

\section{Tissue Preparation}

Tissue samples were resected en bloc from the right and left upper molar regions and fixed in periodic-lysine paraformaldehyde solution at $4{ }^{\circ} \mathrm{C}$ for $24 \mathrm{~h}$. The samples were cut into two slices, which included the first or the second molars, respectively, at the bucco-palatal plane parallel to each distopalatal root. Slices were then decalcified in $1 \mathrm{mM}$ PBS $(\mathrm{pH}$ 7.4) containing $10 \%$ EDTA for 10 days at $4{ }^{\circ} \mathrm{C}$ and subsequently embedded in paraffin, and serial sections $(4.5 \mu \mathrm{m}$ thick) were cut in the direction parallel to the long axis of the tooth, including root apex and mounted on glass slides. The sections were then stained with hematoxylin and eosin (H\&E) for histological examination. To examine the distribution of bLF, various organs including intestinal tract, spleen, femur, etc were collected.

Some gingival tissues from the animals were stored at $-80^{\circ} \mathrm{C}$ until used for examination of TNF- $\alpha$ and IL- $1 \beta$ mRNA expression.

\section{Immunohistochemistry}

To investigate the expression of TNF- $\alpha$ in the periodontal tissues and distribution of bLF in periodontal and other tissues, the following immunohistochemical staining was performed using the high polymer (HISTOFINE simple stain) method. After dewaxing and rehydration, the sections were incubated in $0.3 \%$ hydrogen peroxide in methanol for $60 \mathrm{~min}$ at room temperature to quench endogenous peroxidase activity. After incubation with protein block for $30 \mathrm{~min}$ at room temperature, they were incubated with polyclonal anti-TNF- $\alpha$ antibody (1:100 dilution) (Santa Cruz Biotechnology, CA, USA) or 1:100 dilution of monoclonal antibLF antibody (Hycult Biotechnology B.V.) at $4^{\circ} \mathrm{C}$ overnight. After twice in PBS for $5 \mathrm{~min}$ each, sections were incubated with HISTOFINE simple stain MAX-PO for $30 \mathrm{~min}$. The color was developed with $0.025 \% 3,3^{\prime}$-diaminobenzidine tetrahydrocloride in TRIS-HCC buffer plus hydrogen peroxide and counterstained with hematoxylin, dehydrated, cleared, and mounted.

\section{TRAP Staining}

TRAP activity was examined for characteristics of osteoclast lineage cells according to the method of Minkin. ${ }^{23}$ The TRAP staining solution containing $50 \mathrm{mM}$ sodium tartrate (Wako, Osaka, Japan) detects osteoclasts specifically. Counterstaining was performed with methyl green solution. Negative staining was performed without substrate.

\section{Histomorphometric Analysis}

For histomorphometric analysis, $>12$ representative specimens including the apex of disto-palatal root of right and left 
upper first molars stained for TRAP activity from each experimental group (six rats) were selected. Osteoclasts appeared along the alveolar bone margin were counted with $1 \mathrm{~mm}$ height from the alveolar crest. TRAP-positive cells with two or more nuclei were defined as osteoclasts and those with mononucleus were defined as preosteoclasts and counted separately.

\section{Statistical Analysis}

The data from osteoclast formation assay and animal experiment are presented as mean \pm s.d. We used a Scheffe's test to check for differences in the numbers of osteoclasts between the three groups in each time. A $P<0.05$ was considered significant.

\section{RESULTS}

OBs Expressed Low-Density Lipoprotein-Related Protein 1

As bLF binds to both lipoprotein-related protein (LRP) 1 and LRP2, we examined their expression. All OB cell lines examined expressed LRP1 but not LRP2 (Figure 1a).

\section{bLF is Endocytosed by Osteoblastic Cells}

LRP1 is known to be an endocytic receptor, therefore, to show internalization of bLF by OBs, uptake of bLF was investigated. ST2 cells incubated with bLF showed positive staining for bLF after $4 \mathrm{~h}$ on the cell membrane and cytoplasm (Figure 1b). The cellular immunolocalization of bLF was detected at $2 \mathrm{~h}$, it rapidly decreased at $8 \mathrm{~h}$ and could not detect at $24 \mathrm{~h}$.

\section{bLF Inhibits LPS-Induced Osteoclastogenesis through OBs}

The inhibitory effects of bLF on LPS-induced cytokine expression in OBs were also examined. At $2 \mathrm{~h}$ after LPS addition, TNF- $\alpha$ mRNA was markedly upregulated and then gradually decreased. RANKL-mRNA expression was also upregulated at 12 and $24 \mathrm{~h}$ after LPS addition, whereas OPG-mRNA expression was downregulated in ST2 cells. In bLF pretreatment group, LPS induced upregulation of TNF- $\alpha$ mRNA expression at $2 \mathrm{~h}$ but the level was lower than that of control. After $4 \mathrm{~h}, \mathrm{TNF}-\alpha$-mRNA level in bLF group was downregulated. Pretreatment with bLF suppressed the LPS-induced upregulation of RANKL mRNA and eliminated downregulation of OPG mRNA by LPS (Figure 1c). Quantitative real-time RT-PCR analysis showed that bLF pretreatment caused $74.6 \%$ downregulation of TNF- $\alpha$-mRNA level induced with LPS stimulation (Figure 1d).

A.a.-LPS stimulated TRAP-positive cell formation in the co-culture of primary $\mathrm{OBs}$ and BMCs. The number of TRAP-positive preosteoclasts and osteoclasts induced by LPS stimulation were suppressed by bLF in a dose-dependent manner (Figure 1e). a

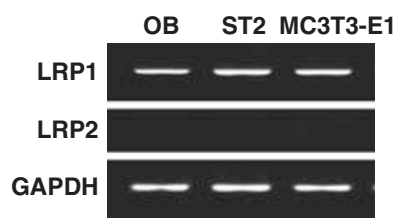

b
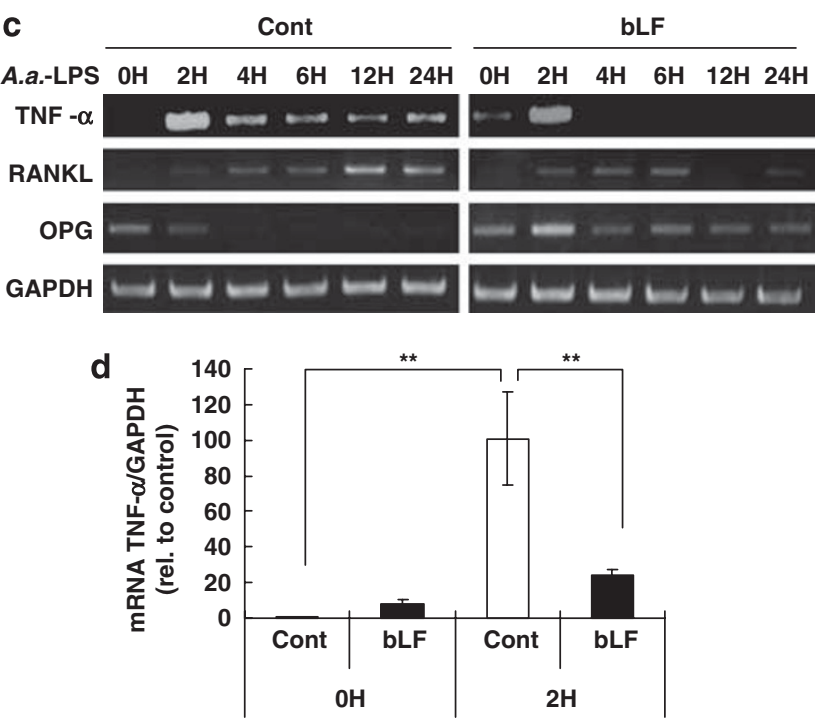

e

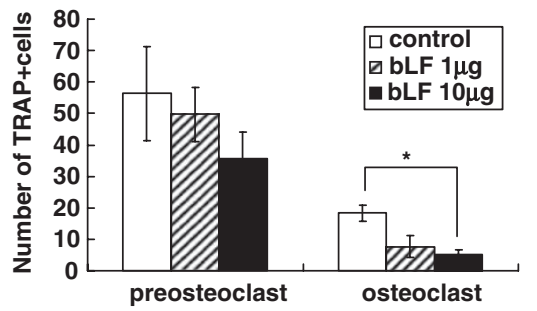

Figure 1 (a) RT-PCR analysis for LRP1 and LRP2 in osteoblast cell lines. GAPDH was used to control. (b) Immunolocalization of bLF in ST2 cells at $4 \mathrm{~h}$ after bLF treatment. Scale bar: $10 \mu \mathrm{m}$. (c) Expression of TNF- $\alpha$, RANKL, and OPG mRNAs in ST2 cells. Cells were harvested at various times $(0-24 \mathrm{~h})$ after $100 \mathrm{ng} / \mathrm{ml}$ A.a.-LPS stimulation, with 2-h-pretreatment $(10 \mu \mathrm{g} / \mathrm{ml})$ or without pretreatment (vehicle) of bLF. GAPDH was used to control. (d) Quantitative real-time RT-PCR analysis for TNF- $\alpha$ in osteoblast cell lines. GAPDH was used to control. Results are expressed as mean \pm s.d. ${ }^{* * P}<0.01$. (e) Effect of bLF on in vitro TRAP + cells formation. Primary osteoblasts and BMCs were co-cultured for 5 days. Co-cultures were incubated in the presence of A.a.-LPS $(1 \mu \mathrm{g} / \mathrm{ml})$ for the final 3 days. Pretreatment of bLF ( 1 or $10 \mu \mathrm{g} / \mathrm{ml}$ ) significantly decreased the number of TRAP + preosteoclasts and osteoclasts. Data are present as the means \pm s.d. ( $n=6$ for each group). ${ }^{\star} P<0.05$.

\section{Distribution of Orally Administrated bLF in Periodontal Tissue and Other Tissue}

To examine the distribution of bLF orally administrated, localization of bLF was immunohistochemically examined. A strong positive reaction to bLF was observed in the epithelial cells of the intestinal tract (Figure 2a). Many bLF-positive cells were present in Peyer's patch and the spleen (data not shown). In addition, bLF-positive cells were present in the distal epiphyseal region of the femur, such as OBs lining 
surface of the bone trabeculae, osteocytes, osteoclasts, and chondrocytes (Figure $2 \mathrm{c}$ and d). Odontoblasts in the pulpal chamber, cementoblasts located along tooth root surface, OBs and some periodontal fibroblasts were also positive for bLF (Figure 2e). Although small number of bLF-positive cells was detected in bLF group, the staining intensity was remarkably reduced compared with that in control group (data not shown).

\section{Oral Administration of L-bLF Decreased Number of Osteoclasts Induced by Topically Applied LPS}

LPS-untreated animals with 7-day administration of each drinking water showed only a few osteoclasts along the alveolar bone surface (Figure 3a, d, and g). LPS application caused a marked increase in osteoclasts along the alveolar bone margin at $3 \mathrm{~h}$, which decreased after 1 day in the control group. But osteoclasts increased again at day 3 and the alveolar bone margin adjacent to the periodontal ligament
(PDL) showed an irregular shape due to bone resorption (Figure 3b). Osteoclasts numbers decreased and returned to normal range by day 7 . In contrast, the number of osteoclast showed no marked changes in the bLF and L-bLF groups (Figure $3 \mathrm{e}$ and $\mathrm{h}$ ). Some specimens from the bLF group showed that the number of osteoclasts slightly increased on day 3 .

To identify osteoclasts and preosteoclasts more clearly, we performed TRAP staining on the same split sections. At $3 \mathrm{~h}$ and 3 days after the LPS treatment, large numbers of TRAPpositive osteoclasts and preosteoclasts were found in the control group (Figure $3 \mathrm{c}$ ), whereas markedly smaller number of TRAP-positive cells was seen in the two bLF groups (Figure $3 \mathrm{f}$ and i). The histomorphometric analysis revealed that the numbers of TRAP-positive osteoclasts and preosteoclasts in the untreated control were $5.5 \pm 1.0$ and $8.3 \pm 0.8$, respectively. LPS application caused a biphasic increase in osteoclasts (Figure 4a) and preosteoclasts
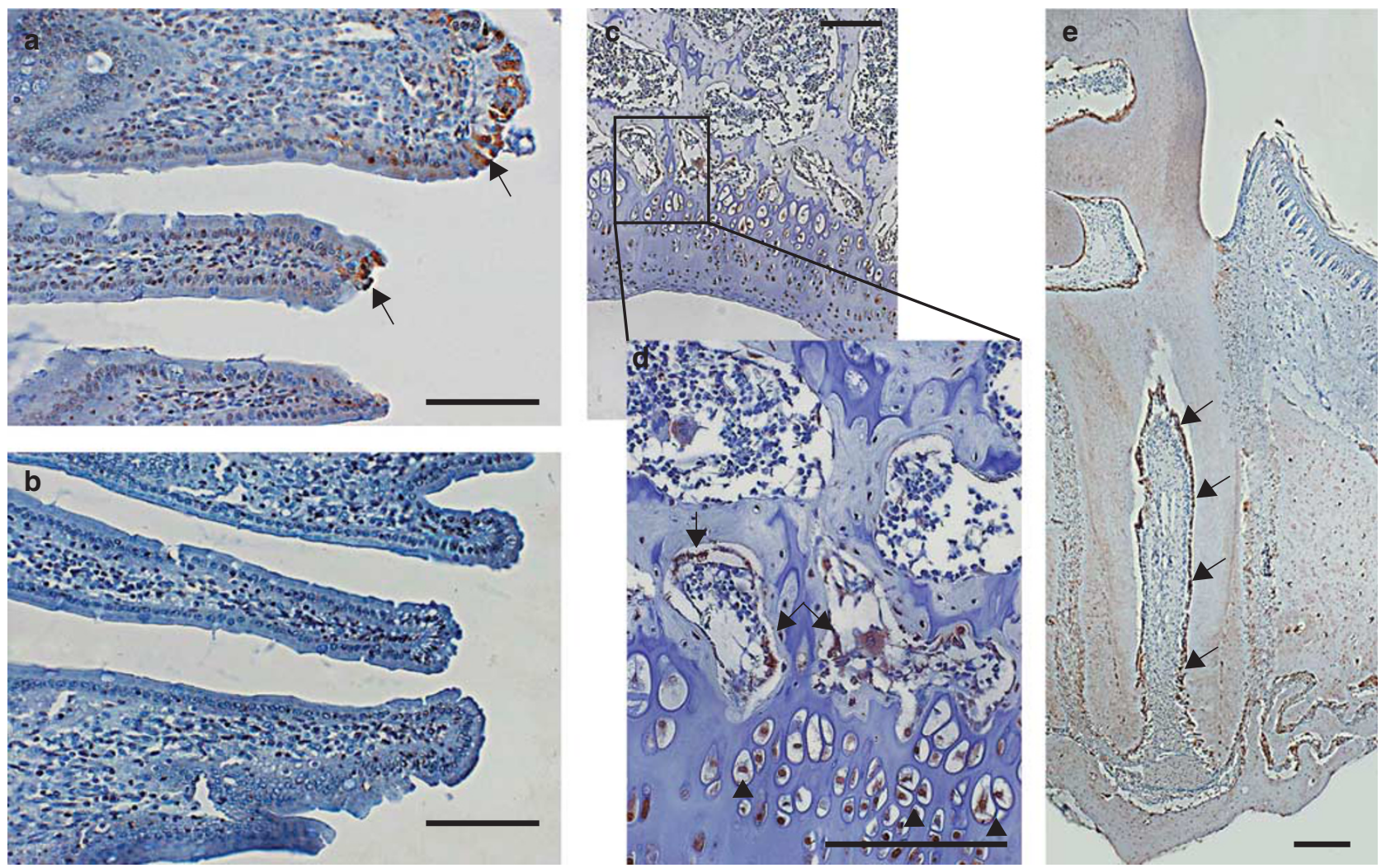

Figure 2 Immunodistribution of bLF absorbed from intestinal tract in various rat organs. (a) Intestinal tract. Some intestinal epithelial cells $(\rightarrow)$ were positively stained for bLF. (b) Negative control. (c, d) Epiphyseal region of femur. Numerous osteoblasts $(\rightarrow)$ lined surface of bone trabeculae and chondrocytes $(\boldsymbol{\Lambda})$ were intensely positive for bLF. (e) Periodontal area. In addition to fibroblasts, osteoblasts, cementoblasts, odontoblasts ( $\rightarrow$ ) were positive for bLF. Scale bars: $100 \mu \mathrm{m}$.

Figure 3 Effects of bLF on alveolar bone destruction caused by LPS application. (a-c) Control group, (a) without LPS, H\&E staining, (b, c) at 3 days after LPS application, (b) H\&E staining, (c) TRAP staining. Numerous osteoclasts were seen along alveolar bone margin. (d-f) bLF group (intake of $470.4 \mathrm{mg} / \mathrm{kg} / \mathrm{day}$ ), (d) without LPS, H\&E staining, (e, f) at 3 days after LPS application, (e) H\&E staining, (f) TRAP staining. (g-i) L-bLF group (intake of 460.4 mg/kg/day), (g) without LPS, H\&E staining, (h, i) at 3 days after LPS application, (h) H\&E staining, (i) TRAP staining. In both bLF groups, bLF treatment suppressed the osteoclast induction caused by LPS application. Particularly, the inhibition in L-bLF group is prominent. Scale bars: $100 \mu \mathrm{m}$. 

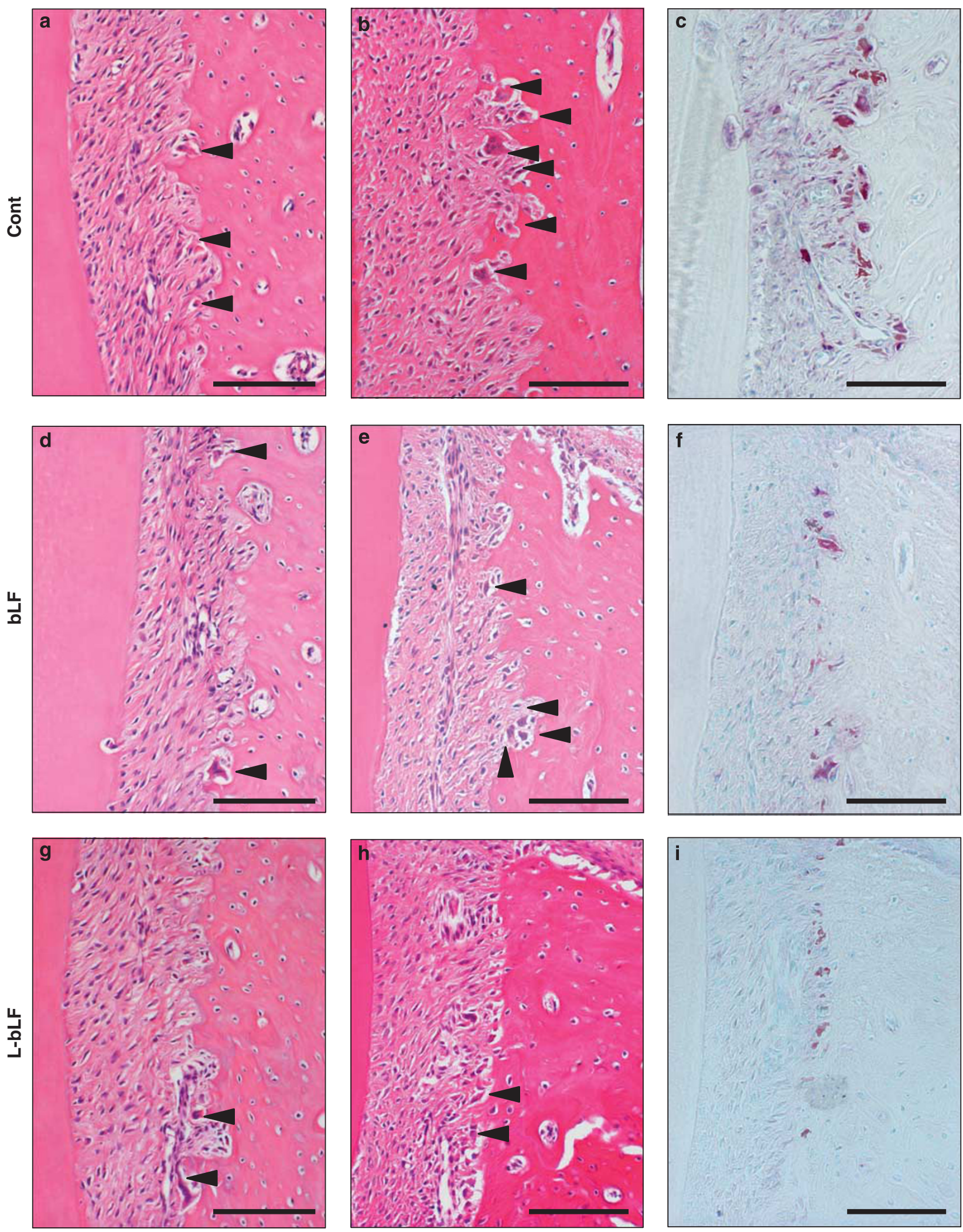

LPS(-)

LPS(+) 3D 
(Figure 4b) peaking at $3 \mathrm{~h}$ (osteoclasts; $8.8 \pm 2.28$, preosteoclasts; $12.6 \pm 3.57, P<0.05$ ) and 3 days (osteoclasts; $12.0 \pm 1.0$, preosteoclasts; $20.0 \pm 3.60, P<0.01)$ compared with the untreated control group $(5.5 \pm 1.0)$. The number of TRAP-positive osteoclasts and preosteoclasts in the two bLF groups was lower than the control group. Especially, the number of osteoclasts in the L-bLF group was significantly inhibited $(P<0.01)$ at $3 \mathrm{~h}(5.0 \pm 1.41)$ and 3 days $(4.0 \pm 1.82)$.

\section{Oral Administration of L-bLF Reduces Upregulation of TNF- $\alpha$ Induced by Topically Applied LPS}

Immunohistochemical staining of TNF- $\alpha$ in the gingival tissue and PDL in each group is shown in Figure 4a. In untreated control group, there is no obvious TNF- $\alpha$
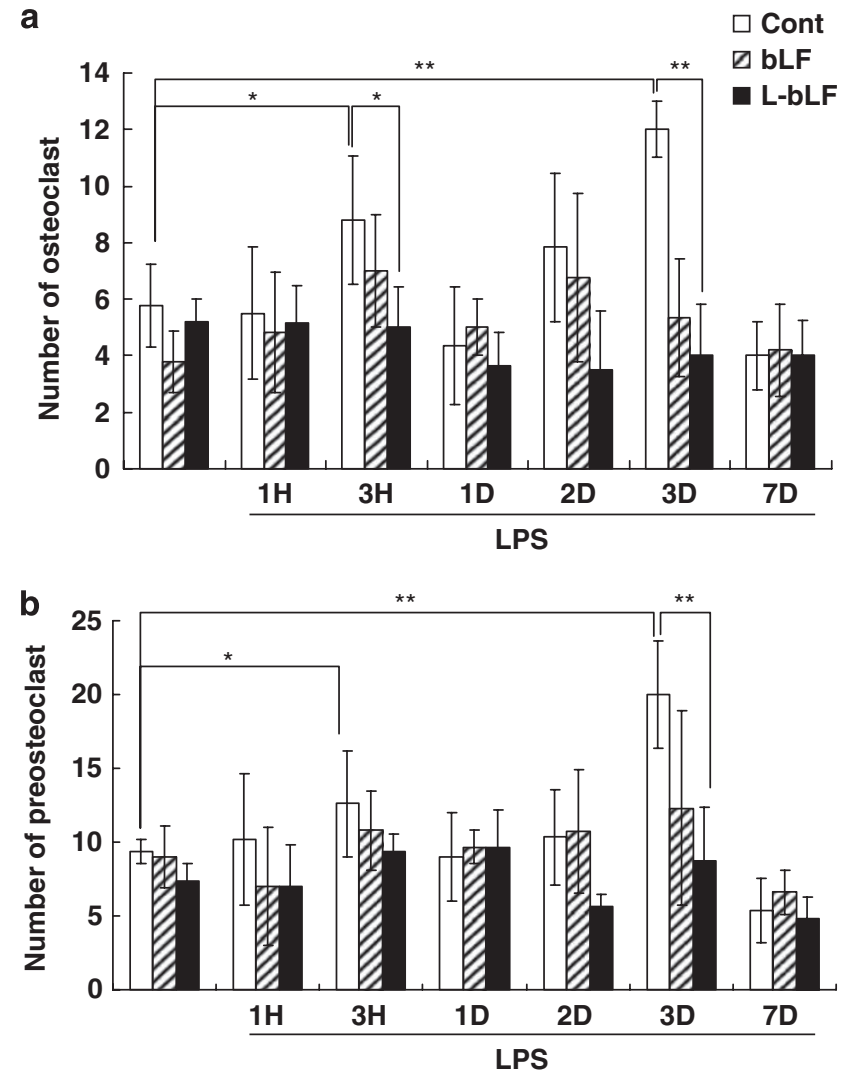

Figure 4 Effects of bLF on the number of TRAP-positive cells appeared along alveolar bone surface. (a) TRAP-positive cells with three or more nuclei were defined as osteoclasts. (b) TRAP-positive cells with two or less nuclei were defined as preosteoclasts. Data are mean \pm s.d. ${ }^{*} P<0.05$, ${ }^{* *} P<0.01$. immunolocalization in gingival tissue (Figure 5aA) and PDL (Figure $5 \mathrm{aB}$ ). LPS induced TNF- $\alpha$ expression in both gingival tissue and PDL at $3 \mathrm{~h}$ (Figure $5 \mathrm{aC}$ and $\mathrm{D}$ ). TNF- $\alpha$ expression decreased but was still present in the JE and PDL areas after 3 days of LPS application in control group. In the L-bLF group, the number of TNF- $\alpha$-positive cells was smaller in the JE (Figure $5 \mathrm{aE}$ ), but not in the PDL (Figure $5 \mathrm{aF}$ ) at $3 \mathrm{~h}$ after LPS application. TNF- $\alpha$-positive cells were not detected in the JE and PDL in the L-bLF group at 3 days after LPS application. The immunoexpression of TNF- $\alpha$ in bLF group was at $3 \mathrm{~h}$ after LPS application was detected, but its intensity was remarkably reduced (data not shown).

TNF- $\alpha$-mRNA expression in the gingival tissue at 2 days after LPS treatment was markedly inhibited in both bLF administrated groups but especially in the L-bLF group (Figure $5 b$ ). Figure $5 \mathrm{c}$ showed TNF- $\alpha$ and IL- $1 \beta$-mRNA levels in gingival tissue. IL-1 $\beta$-mRNA expression level was significantly downregulated by L-bLF administration $(P<0.05)$. TNF- $\alpha$-mRNA level showed a tendency of downregulation but not significant difference.

\section{DISCUSSION}

bLF not only promotes bone growth by stimulating the proliferation and differentiation of the $\mathrm{OB}^{24}$ but also inhibits osteoclast formation from BMCs stimulated with RANKL. ${ }^{25}$ In this study, we examined the effects of bLF on LPS-induced osteoclastogenesis through OBs using in vitro culture system.

All osteoblastic cells examined in this study, expressed LRP1 but not LRP2. LRP1 and LRP2 are well-known endocytotic receptor. Rat primary OBs express both LRP1 and LRP2, and especially LRP1 might contribute to the internalization of bLF and proliferation through p42/44 MAPK signaling in osteoblastic cells. ${ }^{26}$ On the other hand, mouse and human OBs mainly express LRP1. LRP1 in human OB is responsible for the uptake of dietary lipids like lipophilic vitamin $\mathrm{K} 1 .^{27}$

There are some reports that studied the direct effects of bLF on osteoclastogenesis. Cornish et al ${ }^{28}$ studied the effects of bLF on osteoclastogenesis through OBs and mature osteoclasts. Although exposure of OBs to bLF resulted in downregulation of OPG-mRNA and a transient upregulation of RANKL-mRNA, osteoclastogenesis in mouse bone marrow cultures was dose dependently reduced by bLF. In addition, bLF had no effect on bone resorption by isolated mature osteoclasts. $^{28}$ Therefore, they concluded that bLF inhibited bone resorption by reducing the number of osteoclasts formed from the precursor cells in the bone marrow culture.

Figure 5 Effects of oral administration of bLF on the expression of TNF- $\alpha$. (a) Immunoexpression of TNF- $\alpha$ in the gingival tissue and periodontal ligament (PDL). (A, B) In control group without LPS application, TNF- $\alpha$-positive cells were not detected. (C, D) In control group, TNF- $\alpha$ was intensely positive in JE (C) and PDL (D) at $3 \mathrm{~h}$ after LPS application. (E, F) In the L-bLF group, TNF- $\alpha$-positive cells could not observe in gingival tissue (E) and PDL (F) at $3 \mathrm{~h}$ after LPS application. Scale bars: $100 \mu \mathrm{m}$. (b) TNF- $\alpha$-mRNA expressions in gingival tissue obtained from control and bLF animals at 2 days after LPS application by RTPCR analysis. GAPDH was used to control. (c) Quantitative real-time RT-PCR analyses for TNF- $\alpha$ and IL-1 $\beta$ mRNA in gingival tissue obtained from control and L-bLF animals at 2 days after LPS application. GAPDH was used to control. Results are expressed as mean \pm s.d. ${ }^{\star} P<0.05$ vs control. 

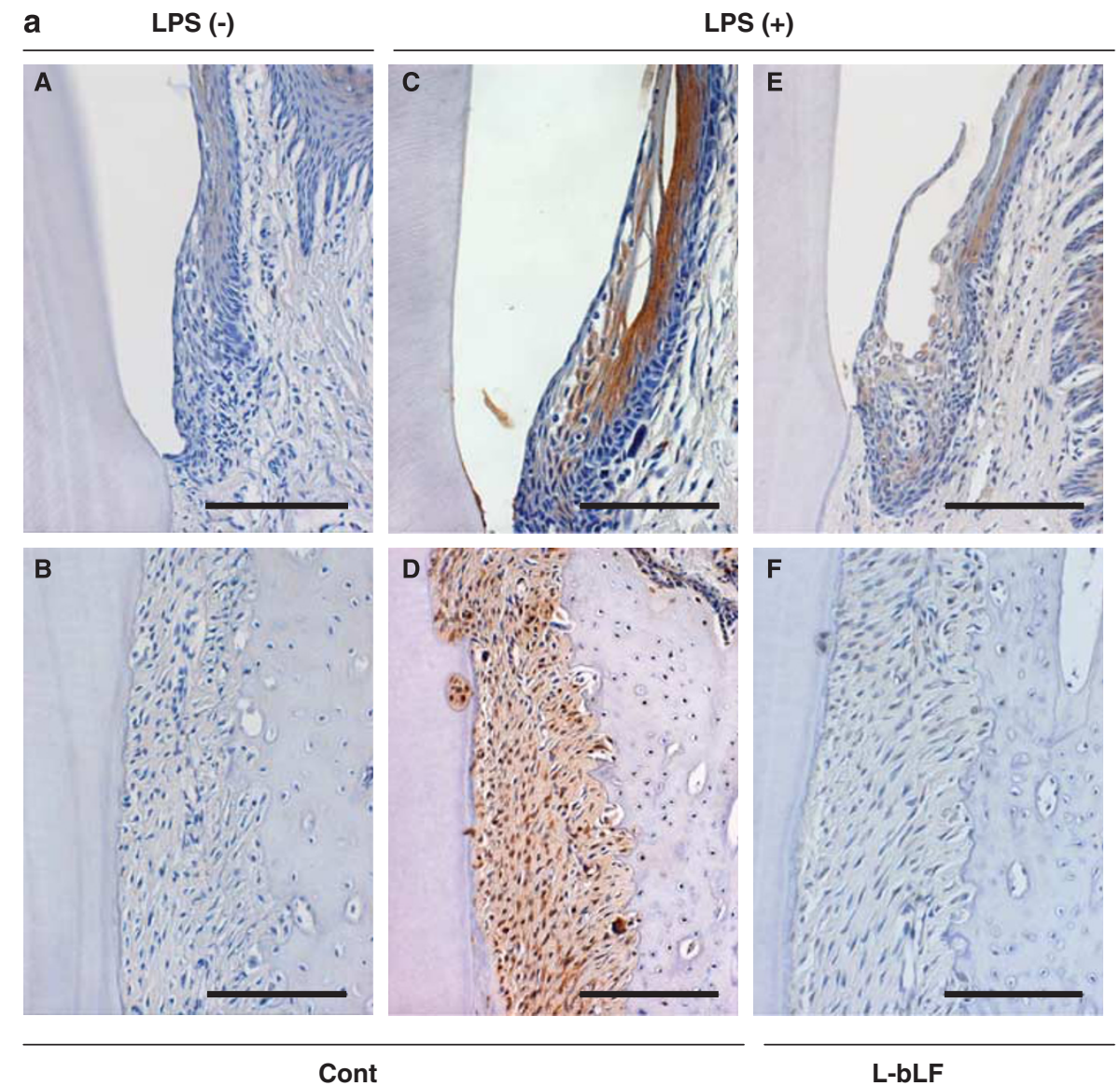

L-bLF

b

LPS(-)
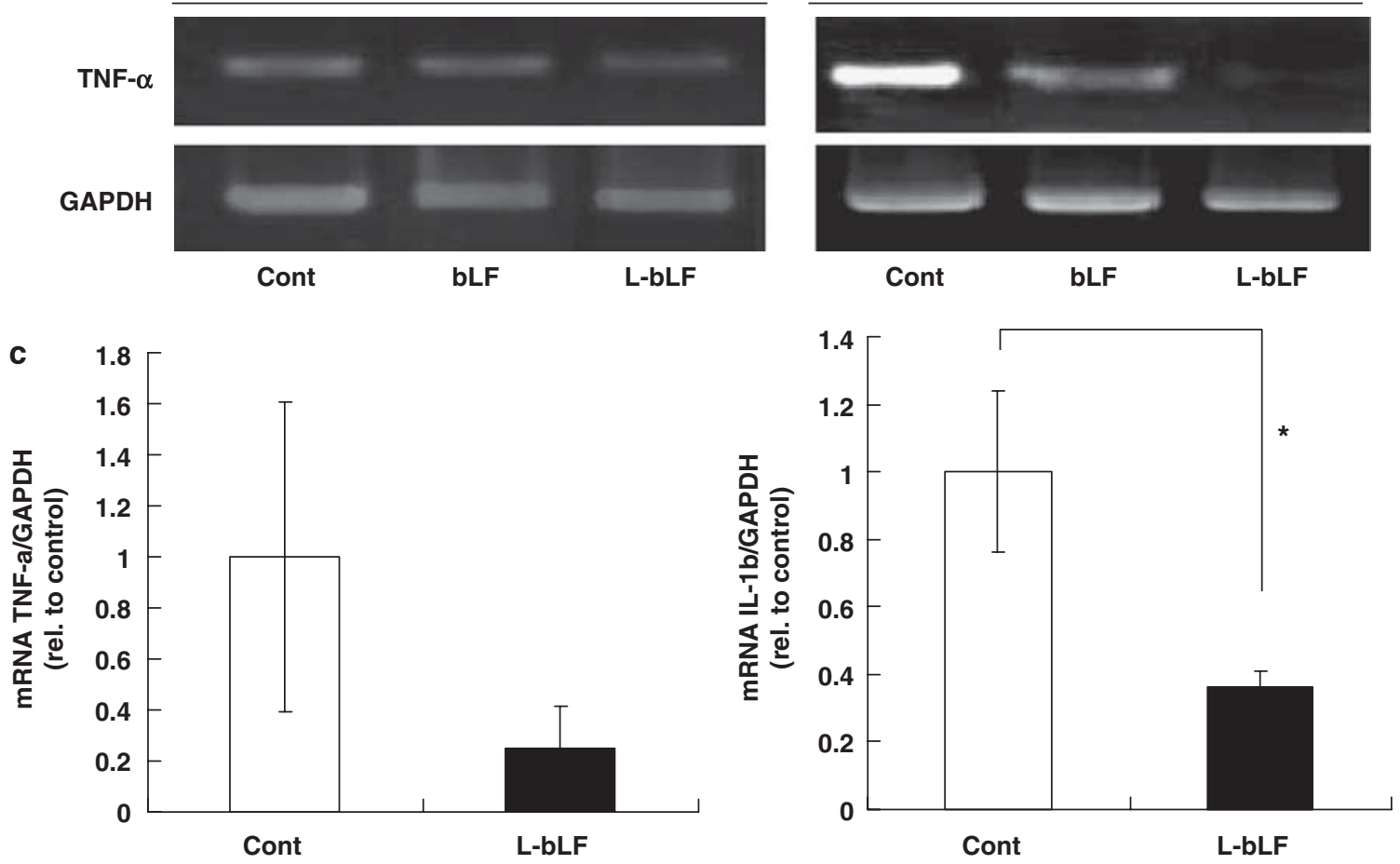
Lorget et $a l^{25}$ also showed that reduced bone resorption by bLF was shown in rabbit-mixed bone cell culture through a mechanism independent of the RANK/RANKL/ OPG system. However, the effect of bLF on osteoclastogenesis induced by LPS has not been reported. We showed that a 2-hpretreatment of bLF caused inhibition of LPS-induced osteoclastogenesis through downregulation of TNF- $\alpha$ and RANKL-mRNA expression and elimination of OPG-mRNA downregulation. Downregulation of TNF- $\alpha$ by bLF possibly can be partially related to bLF binding to LPS, and this may inhibit LPS binding to CD14 by competing with binding of LPS-binding protein to CD14. ${ }^{29-31}$ In this study, extracellular free bLF was removed by washing with PBS before LPS stimulation to avoid formation of bLF-LPS complex. Therefore, we considered that response of OB to LPS may be affected by bLF pretreatment. In fact, LPS-induced TNF- $\alpha$ production by the peripheral monocytes of the mice with orally administrated bLF for 7 days was remarkably reduced. ${ }^{22}$ Recently, it is reported that LPS-induced TNF- $\alpha$ production was suppressed by bLF through its inhibition of binding of NF- $\kappa \mathrm{B}$ to the TNF- $\alpha$ promoter after internalization of bLF in monocytic cell. ${ }^{15}$ It is reasonable to consider that internalized bLF may also reduce LPS-induced TNF- $\alpha$ and RANKL production by inhibiting the binding of NF- $\kappa \mathrm{B}$ to TNF- $\alpha$ promoter in OBs. However, little is known about the intracellular pathways and the role of internalized bLF on $\mathrm{OB}$ functions, which is responsible for osteoclastogenesis. Further studies are needed to clarify the mechanism(s) of bLF-induced inhibition of osteoclastogenesis caused by LPS.

We examined the inhibitory effects of bLF and L-bLF on E. coli LPS $(5 \mathrm{mg} / \mathrm{ml})$-induced periodontal tissue responses, especially increase in osteoclasts and upregulation of TNF- $\alpha$ expression, compared with control group. Although E. coli LPS used to provoke in vivo periodontal tissue damage is not periodontal pathogen, it has the same kind of responses as A.a. LPS. $^{32}$ In addition, periodontal tissue destruction caused by topical application of $E$. coli LPS into the rat gingival sulcus is well established. ${ }^{7-11}$ In our animal model, typical B-cell lesion-like human chronic periodontitis did not develop. However, it has been well accepted that periodontal tissue destruction progresses by recurrent acute episodes. ${ }^{1}$ Therefore, we considered that this could be an useful animal model for the periodontal tissue destruction during acute inflammatory episodes caused by an accumulation of plaqueassociated bacteria.

During experimental period, water consumption and body weight changes in the bLF and L-bLF groups were comparable to control group (data not shown). Body weight in each group was almost the same. No side effects were observed in both of bLF and L-bLF-treated animal. The mean water consumption during the experimental period was $62.3,63.5$, and $62.4 \mathrm{ml}$ in the control, bLF, and L-bLF groups, respectively. The bLF intake calculated from water consumption for the bLF group $(460.4 \mathrm{mg} / \mathrm{kg} /$ day $)$ and L-bLF group $(470.4 \mathrm{mg} / \mathrm{kg} /$ day $)$ were not significantly different.
Oral administration of both forms of bLF inhibited increase in osteoclasts and preosteoclasts with LPS application through suppression of TNF- $\alpha$ production. This is the first study to show preventive and therapeutic effects of bLF on LPS-induced periodontitis.

TNF- $\alpha$ is one of the key cytokines involved in the pathogenesis of periodontitis through inductive production of other proinflammatory cytokines like IL-1 $\beta$, chemokines, and prostaglandin $\mathrm{E}_{2}$. TNF- $\alpha$ also stimulates collagenase production and osteoclastic bone resorption. In our animal model, downregulation of TNF- $\alpha$ produced locally in periodontal tissue, is a critical causative event for inhibiting the increase in osteoclasts and preosteoclasts numbers at 3 days after LPS application. Periodontitis is an infectious disease. However, tissue destruction associated with periodontitis cannot be entirely explained by the action of infecting organisms. The inflammation caused by the periodontal pathogens has a critical function in periodontal tissue destruction. Some reports showed that application of reagents having anti-inflammatory effects not only resolved inflammation but also inhibited tissue and bone loss. ${ }^{33}$ This suggests that drugs with anti-inflammatory effects may offer a novel therapeutic approach for periodontal tissue destruction.

bLF is easily degradated to digestive enzymes such as pepsin or trypsin. About $60 \%$ of the intact bLF enters the small intestine after oral ingestion. ${ }^{19}$ Therefore, we decided to use L-bLF to protect bLF from enzymatic digestion in the stomach. Recently, liposomalization of bLF was shown to improve resistance of bLF to gastric digestion, suggesting that oral administration of L-bLF could be a novel active constituent useful for preventive and therapeutic treatment of inflammatory diseases. ${ }^{20-22}$ Expectedly, oral administration of L-bLF exhibited more stable and effective suppression in the increase of osteoclasts and preosteoclasts than bLF in our animal model. These results suggest that intact bLF absorbed from intestinal mucosa may be important for its inhibitory effects. Further studies are needed to clarify this issue.

Transport routes of orally administrated L-bLF to periodontal tissue remain unclear. In this study, we analyzed the localization of bLF absorbed from the intestinal tract immunohistochemically. bLF-containing cells were distributed not only in the intestinal tract but also in the whole body including spleen, bone, pulpal, and periodontal tissue. It is reported that bLF entered into the blood circulation through the lymphatic pathway after oral administration. ${ }^{34}$ L-bLF transported through the blood circulation may locally affect periodontal tissue cells. In addition, L-bLF in drinking water can also penetrate directly into periodontal tissue through JE and act on its receptors on tissue-resident cells. Moreover, participation of the intestinal tract immunity system of the mesentery lymph node and the Payer's patch has been reported. ${ }^{35}$ Orally administrated bLF stimulated intestineassociated immune functions including the production of IL-18 and IFN and increased NK cell activity. ${ }^{36}$ Macrophages, 
with low ability to produce TNF- $\alpha$, internalized bLF at the Payer's patch, may infiltrate into LPS-applied periodontal tissue through the circulatory system in the L-bLF group. The downregulation of TNF- $\alpha$ production from these macrophages resulted in the inhibition of bone resorption. As mentioned earlier, an increase in the amount of intact bLF entering small intestine by liposomalization had more effective and stable suppression of osteoclastogenesis in this animal model. Therefore, we considered that main bLFtransport route might be blood circulation pathway.

In summary, orally administrated L-bLF can reach the intestinal tract as intact bLF, be absorbed from intestinal epithelial cells, and then be delivered all over the rat's body including periodontal tissue. bLF reached at periodontal tissue reduces inflammation through suppression of TNF- $\alpha$ locally produced from host cells with LPS stimulation. Moreover, bLF may directly inhibit OB-mediated osteoclastogenesis. Overall, the subsequent alveolar bone destruction is reduced in a direct and/or in an indirect manner. These results indicate the possibility of the preventive and therapeutic usage of bLF for LPS-induced periodontitis.

Recently, iron sequestration by bLF was reported to be inhibitory to bacterial biofilm formation. ${ }^{37}$ L-bLF can be used for preventing periodontitis by inhibiting plaque formation. Moreover, accumulating evidence indicates that not only systemic disease affected periodontal condition but also the periodontitis is a potential risk factor for increased morbidity and mortality for several systemic diseases including cardiovascular, pregnancy complications, and diabetes mellitus. ${ }^{38}$ In particular, it is well documented that the severity of chronic periodontitis is positively associated with plasma TNF- $\alpha$ levels in type 2 diabetic patients. ${ }^{39}$ TNF- $\alpha$ produced from the adipocytes was shown to be a pathogenic factor linking obesity to diabetes and periodontal disease. ${ }^{40}$ These findings indicate that systemic approach for periodontal therapy and prevention are needed in addition to the local control of infection and inflammation. Therefore, we suggest that orally administrated L-bLF may be more effective for the prevention of periodontitis especially in patients with diabetes mellitus.

Oral administration of L-bLF could regulate connective tissue destruction and alveolar bone resorption by blocking the first step of proinflammatory cytokine network through inhibition of LPS-induced TNF- $\alpha$ production from host cells.

Supplementary Information accompanies the paper on the Laboratory Investigation website (http://www.laboratoryinvestigation.org)

\section{DISCLOSURE/CONFLICT OF INTEREST}

The authors declare no conflict of interest.

1. Socransky SS, Haffajee AD. The bacterial etiology of destructive periodontal disease: current concepts. J Periodontol 1992;63: 322-331.

2. Wilson M, Reddi K, Henderson B. Cytokine-inducing components of periodontopathgenic bacteria. J Periodont Res 1996;31:393-407.
3. Masada MP, Persson R, Kenney JS, et al. Measurement of interleukin- $1 \alpha$ and $-1 \beta$ in gingival crevicular fluid: implications for the pathogenesis of periodontal disease. J Periodont Res 1990;25:156-163.

4. Reinhardt RA, Masada MP, Kaldahl WB. Gingival fluid IL-1 and IL-6 levels in refractory periodontitis. J Clin Periodontol 1993;20: 225-231.

5. Rossomando EF, Kennedy JE, Hadjimichael J. Tumor necrosis factor- $\alpha$ in gingival crevicular fluid as a possible indicator of periodontal disease in humans. Arch Oral Biol 1990;35:431-434.

6. Katagiri T, Takahashi N. Regulatory mechanisms of osteoblast and osteoclast differentiation. Oral Dis 2002;8:147-159.

7. ljuhin N. Light and electron microscopic studies of experimentallyinduced pathologic changes in the rat periodontal tissue. Adv Dent Res 1988;2:209-214.

8. Miyauchi $M$, Takata $\mathrm{T}$, Ito $\mathrm{H}$, et al. Distribution of macrophage lineage cells in rat gingival tissue after topical application of lipopolysaccharide: an immunohistochemical study using monoclonal antibodies: OX6, ED1 and ED2. J Periodont Res 1998;33:345-351.

9. Takata T, Miyauchi M, Ogawa I, et al. Reactive change in proliferative activity of the junctional epithelium after topical application of lipopolysaccharide. J Periodontol 1997;68:531-535.

10. Miyauchi $M$, Sato $S$, Kitagawa $S$, et al. Cytokine expression in rat molar gingival periodontal tissues after topical application of lipopolysaccharide. Histochem Cell Biol 2001;116:57-62.

11. Miyauchi M, Kitagawa S, Hiraoka M, et al. Immunolocalization of CXC chemokine and recruitment of polymorphonuclear leukocytes in the rat molar periodontal tissue after topical application of lipopolysaccharide. Histochem Cell Biol 2004;121:291-297.

12. Brock JH. Lactoferrin: a multifunctional immunoregulatory protein? Immunol Today 1995;16:417-419.

13. Damiens E, Mazurier J, Yazidi I, et al. Effects of human lactoferrin on NK cell cytotoxicity against haematopoietic and epithelial tumour cells. Biochim Biophys Acta 1998;1402:277-287.

14. Choe YH, Lee SW. Effect of lactoferrin on the production of tumor necrosis factor-alpha and nitric oxide. J Cell Biochem 1999;76: 30-36.

15. Haversen L, Ohlsson BG, Hahn-Zoric M, et al. Lactoferrin downregulates the LPS-induced cytokine production in monocytic cells via NF- $\kappa$ B. Cell Immunol 2002;220:83-95.

16. Guillen $C$, Mclnnes IB, Vaughan $D$, et al. The effects of local administration of lactoferrin on inflammation in murine autoimmune and infectious arthritis. Arthritis Rheum 2000;43: 2073-2080.

17. Hayashida K, Kaneko T, Takeuchi T, et al. Oral administration of lactoferrin inhibits inflammation and nociception in rat adjuvantinduced arthritis. J Vet Med Sci 2004;66:149-154.

18. Wei PF, Ho KY, Ho YP, et al. The investigation of glutathione peroxidase, lactoferrin, myeloperoxidase and interleukin-1beta in gingival crevicular fluid: implications for oxidative stress in human periodontal diseases. J Periodont Res 2004;39:287-293.

19. Troost FJ, Steijns J, Saris WH, et al. Gastric digestion of bovine lactoferrin in vivo in adults. J Nutr 2001;131:2101-2104.

20. Ishikado A, Imanaka $\mathrm{H}$, Kotani $\mathrm{M}$, et al. Liposomal lactoferrin induced significant increase of the interferon-alpha (IFN-alpha) producibility in healthy volunteers. Biofactors 2004;21:69-72.

21. Trif $M$, Guillen C, Vaughan DM, et al. Liposomes as possible carriers for lactoferrin in the local treatment of inflammatory diseases. Exp Biol Med 2001;226:559-564.

22. Ishikado A, Imanaka $H$, Takeuchi $T$, et al. Liposomalization of lactoferrin enhanced it's anti-inflammatory effects via oral administration. Biol Pharm Bull 2005:28:1717-1721.

23. Minkin $\mathrm{C}, \mathrm{Yu} \mathrm{XH}$. Calcitonin receptor expression and its regulation by 1 alpha-25-dihydroxyvitamin D3 during de novo osteoclast formation in organ cultures of fetal mouse metatarsals. Bone Miner 1991;13:191-200.

24. Cornish J. Lactoferrin promotes bone growth. Biometals 2004;17: 331-335.

25. Lorget $\mathrm{F}$, Clough J, Oliveira $\mathrm{M}$, et al. Lactoferrin reduces in vitro osteoclast differentiation and resorbing activity. Biochem Biophys Res Commun 2002;296:261-266.

26. Grey $A$, Banovic $T$, Zhu $Q$, et al. The low-density lipoprotein receptorrelated protein-1 is a mitogenic receptor for lactoferrin in osteoblastic cells. Mol Endocrinol 2004;18:2268-2278. 
27. Niemeier A, Kassem M, Toedter K, et al. Expression of LRP1 by human osteoblasts: a mechanism for the delivery of lipoproteins and vitamin K1 to bone. J Bone Miner Res 2005;20:283-293.

28. Cornish J, Callon KE, Naot D, et al. Lactoferrin is a potent regulator of bone cell activity and increases bone formation in vivo. Endocrinology 2004; 145:4366-4374.

29. Elass-Rochard E, Eegrand D, Salmon V, et al. Lactoferrin inhibits the endotoxin interaction with CD14 by competition with the lipopolysaccharide-binding protein. Infect Immun 1998;66:486-491.

30. Baveye S, Elass E, Fernig DG, et al. Human lactoferrin interacts with soluble CD14 and inhibits expression of endothelial adhesion molecules, E-selectin and ICAM-1, induced by the CD14lipopolysaccharide complex. Infect Immun 2000;68:6519-6525.

31. Na YJ, Han SB, Kang JS, et al. Lactoferrin works as a new LPS-binding protein in inflammatory activation of macrophages. Int Immunopharmacol 1996;4:1187-1199.

32. Patil C, Rossa Jr C, Kirkwood KL. Actinobacillus actinomycetemcomitans lipopolysaccharide induces interleukin-6 expression through multiple mitogen-activated protein kinase pathways in periodontal ligament fibroblasts. Oral Microbiol Immunol 2006;21:392-398.

33. Hasturk $H$, Kantarci $A$, Ohira $M$, et al. RvE1 protects from local inflammation and osteoclast mediated bone destruction in periodontitis. FASEB J 2005;220:401-403.
34. Takeuchi $\mathrm{H}$, Matsui $\mathrm{Y}$, Yamamoto $\mathrm{H}$, et al. Mucoadhesive properties of carbopol or chitosan-coated liposomes and their effectiveness in the oral administration of calcitonin to rats. J Control Release 2003;86:235-242.

35. Harada E. Characteristic transfer of colostrum-derived biologically active substances into cerebrospinal fluid via blood in natural suckling neonatal pigs. J Vet Med A 2002;49:358-364.

36. Kuhara T, Yamauchi K, Tamura Y, et al. Oral administration of lactoferrin increases NK cell activity in mice via increased production of IL-18 and type I IFN in the small intestine. J Interferon Cytokine Res 2006;26:489-499.

37. Singh PK. Iron sequestration by human lactoferrin stimulates P. aeruginosa surface motility and blocks biofilm formation. Biometals 2004;17:267-270.

38. Moutsopoulos NM, Madianos P. Low-grade inflammation in chronic infectious diseases. Paradigm of periodontal infection. Ann NY Acad Sci 2006;1088:251-264.

39. Engebretson S, Chertog R, Nichols A, et al. Plasma levels of tumor necrosis factor- $\alpha$ in patients with chronic periodontitis and type 2 diabetes. J Clin Periodontol 2007;34:18-24.

40. Genco RJ, Grossi SG, Ho A, et al. A proposed model linking inflammation to obesity, diabetes, and periodontal infections. J Periodontol 2005;76:2075-2084. 